\title{
CALCULATING THE IONIZATION RATE INDUCED BY GCR AND SCR PROTONS IN EARTH'S ATMOSPHERE
}

\author{
E.A. Maurchev \\ Polar Geophysical Institute \\ Apatity, Russia, maurchev1987@gmail.com
}

\section{Yu.V. Balabin}

Polar Geophysical Institute

Apatity, Russia,balabin@pgia.ru

\section{A.V. Germanenko}

Polar Geophysical Institute

Apatity, Russia, alex.germanenko@gmail.com

\author{
E.A. Mikhalko \\ Polar Geophysical Institute \\ Apatity, Russia,mikhalko@pgia.ru
}

\author{
B.B. Gvozdevsky \\ Polar Geophysical Institute \\ Apatity,Russia,gvozdevsky@pgia.ru
}

\begin{abstract}
This paper explores the applied use of the RUSCOSMICS software package [http://ruscosmics.ru] designed to simulate propagation of primary cosmic ray (CR) particles through Earth's atmosphere and collect information about characteristics of their secondary component. We report the results obtained for proton fluxes with energy distributions corresponding to differential spectra of galactic CR (GCR) and solar CR (SCR) during ground level enhancement (GLE) events GLE65 and GLE67. We examine features of the geometry of Earth's atmosphere, parametrization methods, and describe a primary particle generator. The typical energy spectra of electrons obtained both for GCR and for GLE65 provide information allowing quantitative esti-
\end{abstract}

mation of the SCR contribution to the enhancement of secondary CR fluxes. We also present altitude dependences of ionization rate for GCR and both the GLE events for several geomagnetic cutoff rigidities. The conclusion summarizes and discusses the prospects for future research.

Keywords: cosmic rays, astrophysics, Monte Carlo method, GEANT4, particle physics, numerical simulation.

\section{INTRODUCTION}

One of the important tasks in CR physics is to study propagation of elementary particles in Earth's atmosphere, their effect on the surrounding medium and different systems through successive interactions. Estimation of the CR-induced ionization rate has therefore remained relevant for many years [Bazilevskaya et al., 2008.; Usoskin et al, 2009; Velinov et al., 2017]. Since computational technologies are far advanced today, these studies are carried out using not only traditional experimental methods but also numerical simulation. For these purposes at the CR station Apatity, in addition to the existing monitoring system a software package RUSCOSMICS has been developed [Maurchev, Balabin, 2016; http://ruscosmics.ru] based on a toolkit GEANT4 [Agostinelli et al., 2003; Maurchev et al., 2015]. The developed technique can assess the radiation dose at different altitudes in Earth's atmosphere, using standard, inexpensive, and continuous measurements made at ground neutron monitor stations. This will ensure the radiation safety in airliners, especially during transatlantic flights. In this paper, by simulating propagation of protons through Earth's atmosphere we examine the interaction between the particles of both galactic (GCR) and solar (SCR) cosmic rays during ground level enhancement (GLE) events GLE65 and GLE67. We have obtained numerical characteristics of intensity of fluxes of various secondary CR components (muons, protons, neutrons, electrons, and gamma-quanta), and have calculated ionization rates for a range of geomagnetic cutoff rigidities. Some results of the use of GCR spectra have been described in [Maurchev, Balabin, 2016] and therefore we do not present them here. The model is verified by comparing vertical profiles of count rate obtained from balloons and airbus flights and calculated by simulating propagation of GCR protons through Earth's atmosphere.

\section{METHOD}

At the initial stage, it is necessary to define the geometry of the model used for calculations. This is done through parameterization in such a way that the resulting geometry is not only as close to real physical parameters of matter in Earth's atmosphere as possible, but also is optimized for the calculations. The method is based on the concept of plane geometry when an air column is divided into $N$ layers for which mass, density, temperature, and percentage of chemical elements are determined by the NRLMSISE-00 model [Picone et al., 2007] and then are averaged. In practice, it has been found that for a satisfactory final result inconsistent with verification data within $15 \%$, the optimum value $N=20$ (or $5 \%$ of the total mass of the column). The algorithm of the program that makes the calculation from tabulated data is presented in Figure 1. This process repeats itself $N-1$ times, where $N$ is the number of atmospher- 
ic layers, and for $N$ the calculation is made by simply averaging the remaining tabulated values. Percentage of $\mathrm{H}, \mathrm{He}, \mathrm{O}, \mathrm{N}$, and Ar is calculated as in the case of density - from known data.

Thus, the described approach allows us to optimize calculations by varying the weight percentage depending on the desired accuracy. Parameters $\left(T, \rho, \mathrm{O}_{\%}, \mathrm{~N}_{\%}\right.$, $\mathrm{He}_{\%}, \mathrm{Ar}_{\%}$ ) obtained for $67^{\circ} 34^{\prime} 03^{\prime \prime} \mathrm{N}, 33^{\circ} 23^{\prime} 36^{\prime \prime} \mathrm{E}$ ( Apatity) are shown in Figures 2, 3. Note that for other geographic coordinates density values differ from the above ones; an example of this dependence is presented on the website of the project [http://ruscosmics.ru/ CR.htm]. For more information about the NRLMSISE model read [Picone et al., 2002]. At the second stage, we determine the model source of protons. Its major parameters are the position in space and the probability density of generated particles, which corresponds to the differential energy spectrum. Based on the results of previous calculations, it was decided to use a point realization of the generator, i.e. at the upper boundary of the air column in the central part the source of primary particles is set without specifying its spatial distribution.

This approach provides good statistical accuracy in collecting information about intensity of secondary CR particle fluxes. To specify energy characteristics of GCR protons (in $\mathrm{s}^{-1} \mathrm{~m}^{-2} \mathrm{sr}^{-1} \mathrm{MeV}^{-1}$ ), we employ the formula [GOST 25645.150-90. Galactic cosmic rays. Particle flux model]:

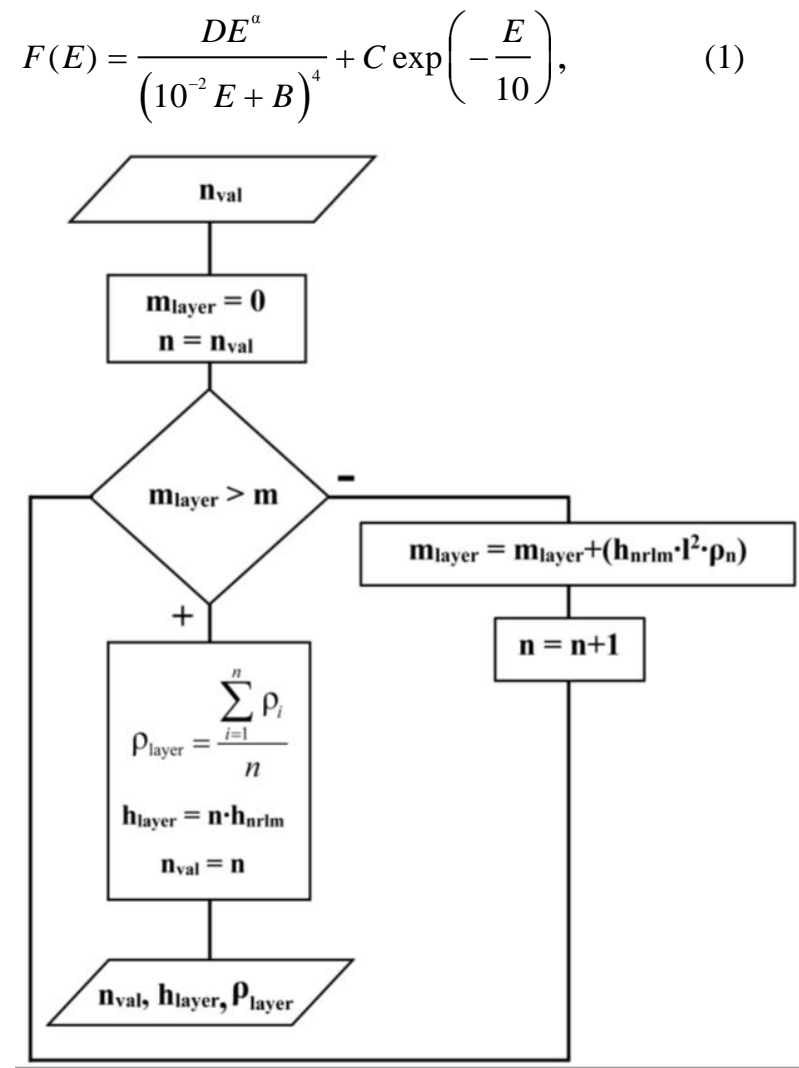

Figure 1. Algorithm for calculating heights and densities for one atmospheric layer in the model of propagation of CR protons: $m_{\text {layer }}$ is the calculated mass of air column from tabulated NRLMSISE values; $M=m / N$ is the mass of the atmospheric layer, $M$ is the mass of the entire air column; $h_{\text {layer }}, h_{\text {nrlm }}$ is the height of layer and the height for tabulated value $(100 \mathrm{~m})$ respectively

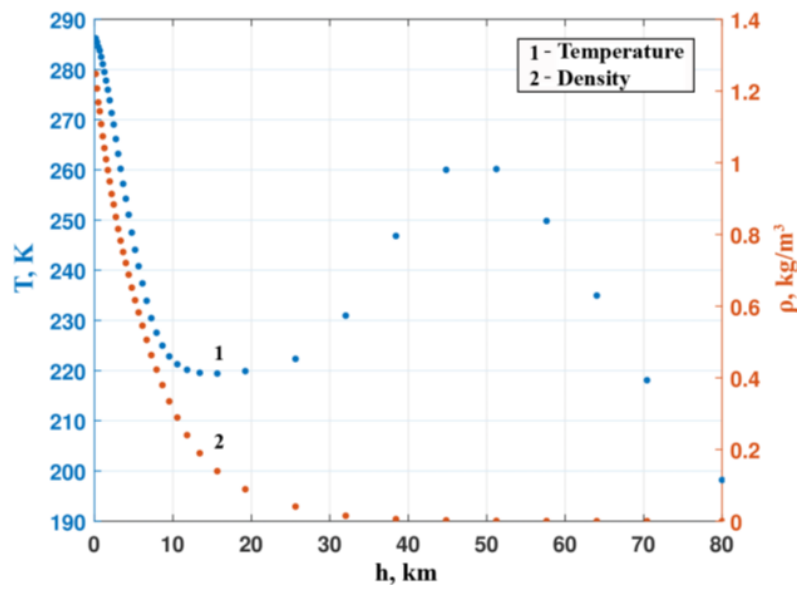

Figure 2. Model parameters of Earth's atmosphere (density and temperature) used to calculate propagation of CR protons through it and to study cascades of secondary particles

where $D, B, C, \alpha$ are the parameters depending on the phase of the 11-year cycle (in this paper, $D=5.2$; $B=6.2 ; \alpha=1.4 ; C=2$, which corresponds to solar minimum). The SCR proton spectrum is described by a model developed at PGI, Apatity [Vashenyuk et al., 2008, 2011; Perez-Peraza et al., 2006, 2008]. According to this model, the spectrum contains two components - fast (PC) and slow (DC); their energy dependences can be expressed $\left(\mathrm{in} \mathrm{s}^{-1} \mathrm{~m}^{-2} \mathrm{sr}^{-1} \mathrm{GeV}^{-1}\right)$ as

$$
\begin{aligned}
& J_{\mathrm{PC}}=J_{0} \exp \left(-E / E_{0}\right) ; \\
& J_{\mathrm{DC}}=J_{1} E^{-\gamma},
\end{aligned}
$$

where $J_{0}, E_{0}, J_{1}, \gamma$ are the coefficients that depend on the number of GLE event. For increases with numbers 65 and 67, parameters listed in Table were employed. Corresponding differential spectra are shown in Figure 4. We can see (Figure 4) that values for GCR and SCR vary up to several orders of magnitude to $5 \mathrm{GeV}$, but starting with $10 \mathrm{GeV}$ the fluxes decrease and total spectra become exactly the same.

Parameters of differential energy spectra of solar protons in GLE65 and GLE67 events

\begin{tabular}{|c|c|c|c|c|c|}
\hline \multirow{2}{*}{$\begin{array}{c}\text { GLE } \\
\text { number }\end{array}$} & \multirow{2}{*}{ Date } & \multicolumn{4}{|c|}{ Parameters of energy spectra } \\
\cline { 3 - 6 } & & $J_{0}$ & $E_{0}$ & $J_{1}$ & $\gamma$ \\
\hline 65 & $\begin{array}{c}\text { October } \\
28,2003\end{array}$ & $1.2 \cdot 10^{4}$ & 0.60 & $1.5 \cdot 10^{4}$ & 4.4 \\
\hline 67 & $\begin{array}{c}\text { November } \\
02,2003\end{array}$ & $4.6 \cdot 10^{4}$ & 0.51 & $9.7 \cdot 10^{3}$ & 6.3 \\
\hline
\end{tabular}

The particle interaction models implemented in GEANT4 are described in detail in the reference manual [GEANT4 Physics Reference Manual]. Depending on information about secondary CR of interest, RUSCOSMICS implemented algorithms able to store datasets as energy spectra and vertical intensity profiles of CRs of different types and ionization rates.

\section{RESULTS}

The simulation of propagation of GCR and SCR protons through Earth's atmosphere has yielded large arrays containing energy spectra of secondary particles and total 

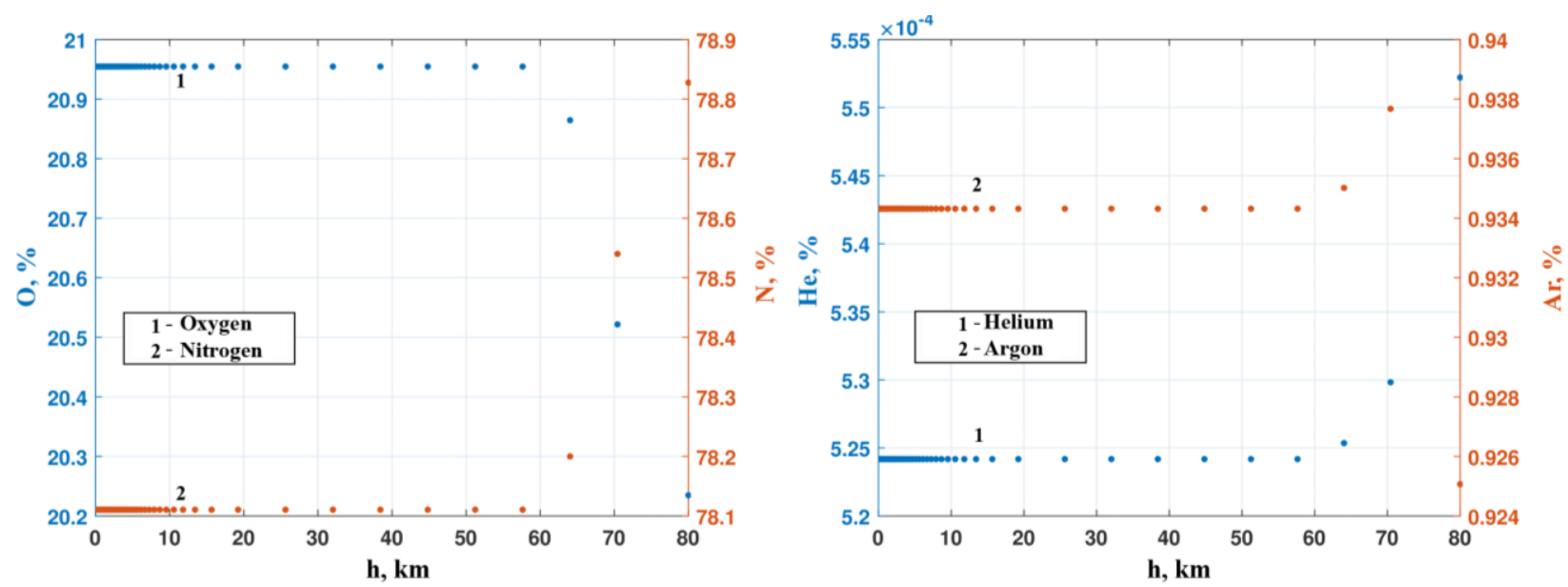

Figure 3. Percentage of elements of Earth's atmosphere derived by averaging. We can see that these parameters weakly depend on altitude, remaining virtually unchanged up to $60 \mathrm{~km}$ above sea level
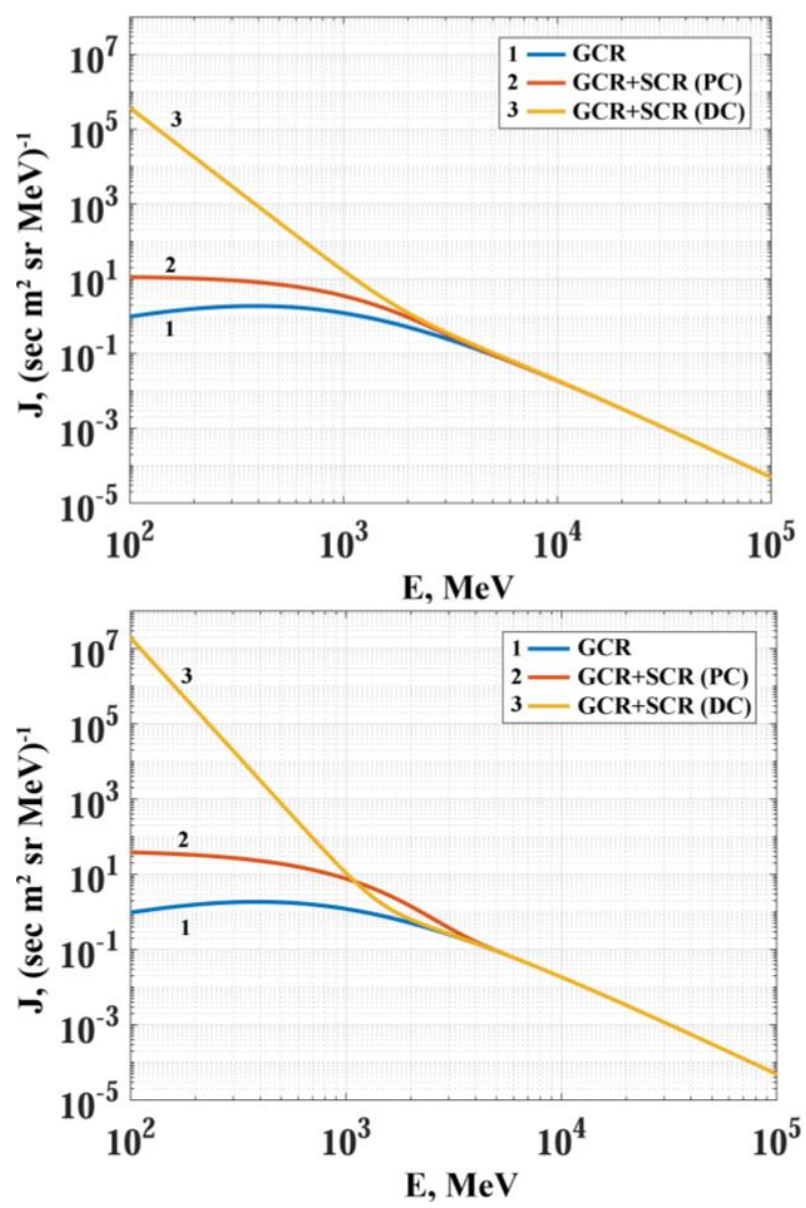

Figure 4. Differential energy spectra of SCR protons from $100 \mathrm{MeV}$ to $100 \mathrm{GeV}$ for GLE65 (a) and GLE67 (b)

total intensities of their fluxes. Figure 5 shows typical distributions only for GCR and GLE65, when GCRs are added to the slow SCR component. We can clearly see (Figure 5) that the fluxes strongly differ to a level of $400 \mathrm{~g} \cdot \mathrm{cm}^{-2}(\sim 7.5 \mathrm{~km}$ above sea level), whereupon, due to the nature of the spectrum of primary particles, they become nearly identical.

Also of particular interest is data on the rate of ionization of substance in Earth's atmosphere by CR particles.
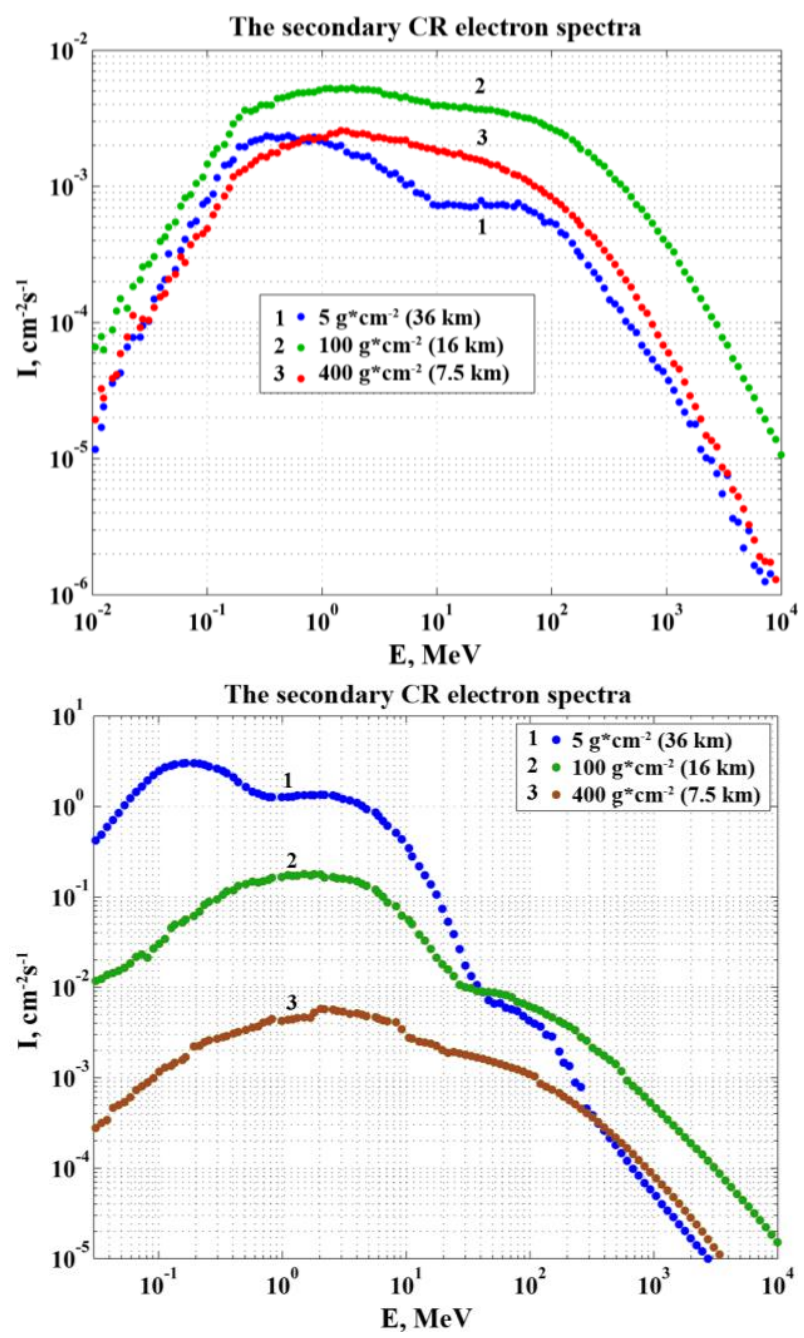

Figure 5. Energy spectra of electrons of secondary GCR (a) and GCR+SCR for the DC component (b) at different atmospheric depths (in brackets is the equivalent altitude)

Model detectors take into account ionization of all types, storing the final result in the format of vertical profiles. The profiles obtained for geomagnetic cutoff rigidities of 0.65 and $2 \mathrm{GV}$ of GCR and SCR protons are shown in Figure 6. 

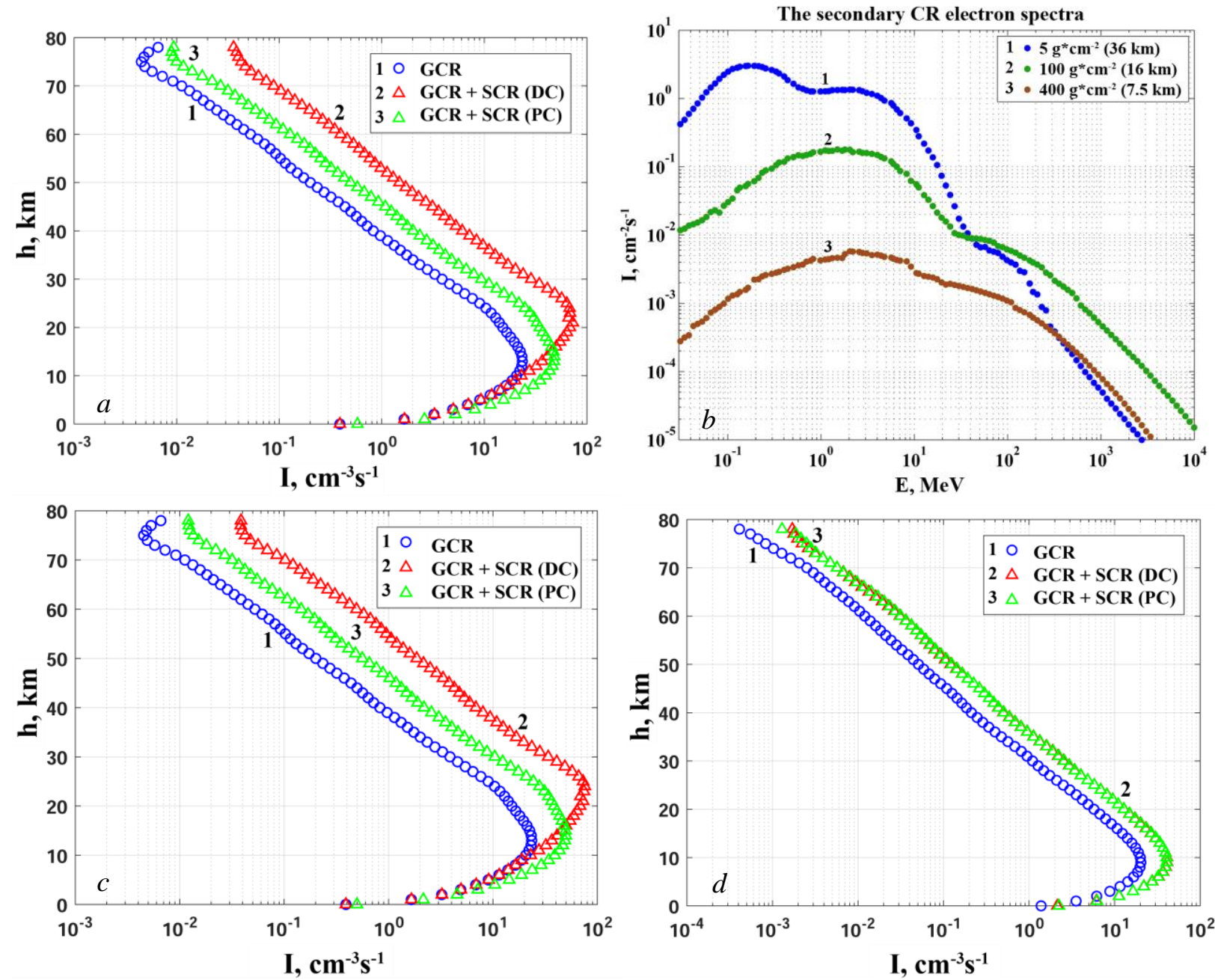

Figure 6. Vertical ionization rate profiles obtained from simulation of propagation of primary CR protons through Earth's atmosphere during $\operatorname{GLE} 65(a, c)$ and $\operatorname{GLE} 67(b, d)$. Geomagnetic cutoff rigidity of $0.65 \mathrm{GV}(a, b)$ and $2 \mathrm{GV}(c, d)$

In addition to the above functions, the RUSCOSMICS software package for simulation of propagation of CR particles through Earth's atmosphere can calculate flux levels and ionization rates for all geographic coordinates. Figure 7 presents the results obtained when the GCR proton spectrum and geomagnetic cutoff rigidities, introduced by IGRF, were utilized as input parameters [Thébault et al., 2015]; the grid spacing is $5^{\circ}$. Detailed datasets for altitudes from 1 to $80 \mathrm{~km}$ are available both in the form of drawings and archives on the website of the project [http://ruscosmics.ru/CR.htm].

\section{VERIFICATION OF CALCULATIONS}

To assess the correctness of our calculations, we use different sets of experimental data. Results of previous comparisons are described in [Maurchev et al., 2018]. For the verification, we use data both from balloons [Stozhkov et al., 2009] and from a portable ionizing-radiation detector developed to expand measurements (Figure 8). This detector was used to record the count rate profile during the flight on the route Mur-
mansk-Apatity. Eight counters STS-6 had been replaced by more compact four counters STS-5, thereby reducing the size of the equipment. Self-contained supply was by a 5 A Li-Ion battery. The total particle flux registered by Geiger counters includes contributions of charged particles and $\sim 1 \%$ of gamma-quanta:

$$
J_{\text {total }}=J_{\mathrm{p}}+J_{\mathrm{e}^{+} \mathrm{e}^{-}}+J_{\mu^{+} \mu^{-}}+0.01 J_{\gamma},
$$

where $J_{\mathrm{p}}$ is the total proton flux; $J_{\mathrm{e}+\mathrm{e}-}$ is the total electron and positron flux; $J_{\mu+\mu-}$ is the total muon flux; $J_{\gamma}$ is the total gamma-quanta flux. Figure 9 compares experimental data obtained from balloons ( Apatity) for several periods corresponding to solar minimum and profiles from the mobile detector with the simulation results. Already at the current stage there is good agreement; a certain difference may be explained by the fact that the model omits helion electrons and elements with $Z>2$. The profiles obtained from airplane measurements were also affected by the difference in periods for which the data was acquired. At present, the work on removing these limitations in the RUSCOSMICS software package is in progress. 

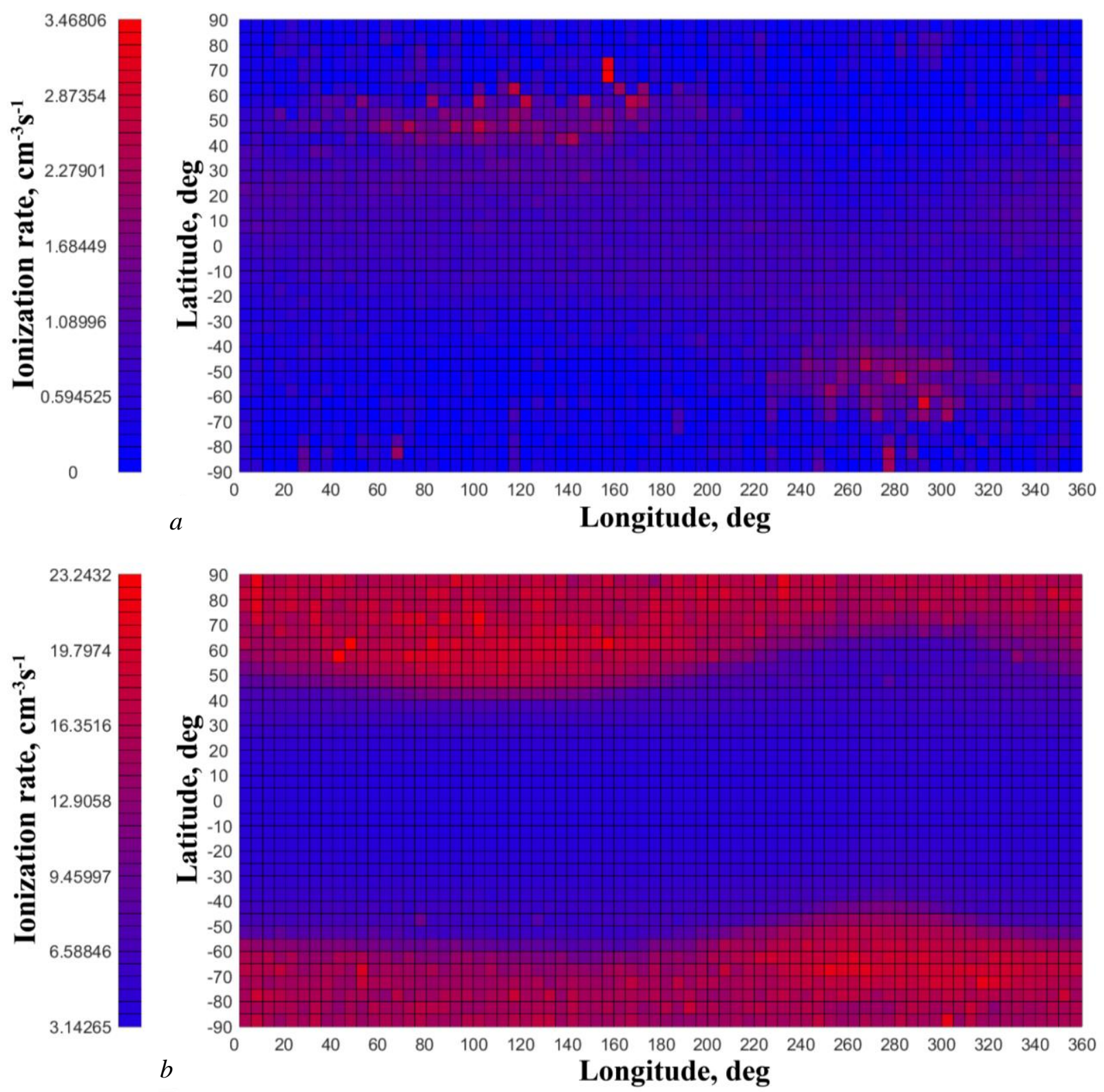

Figure 7. Ionization rates obtained from the simulation of propagation of primary GCR through Earth's atmosphere and the formation of cascades of secondary CRs in it, using the global geometry ( $5^{\circ}$ grid spacing). The data plotted corresponds to the altitudes of $1 \mathrm{~km}(a)$ and $10 \mathrm{~km}(b)$

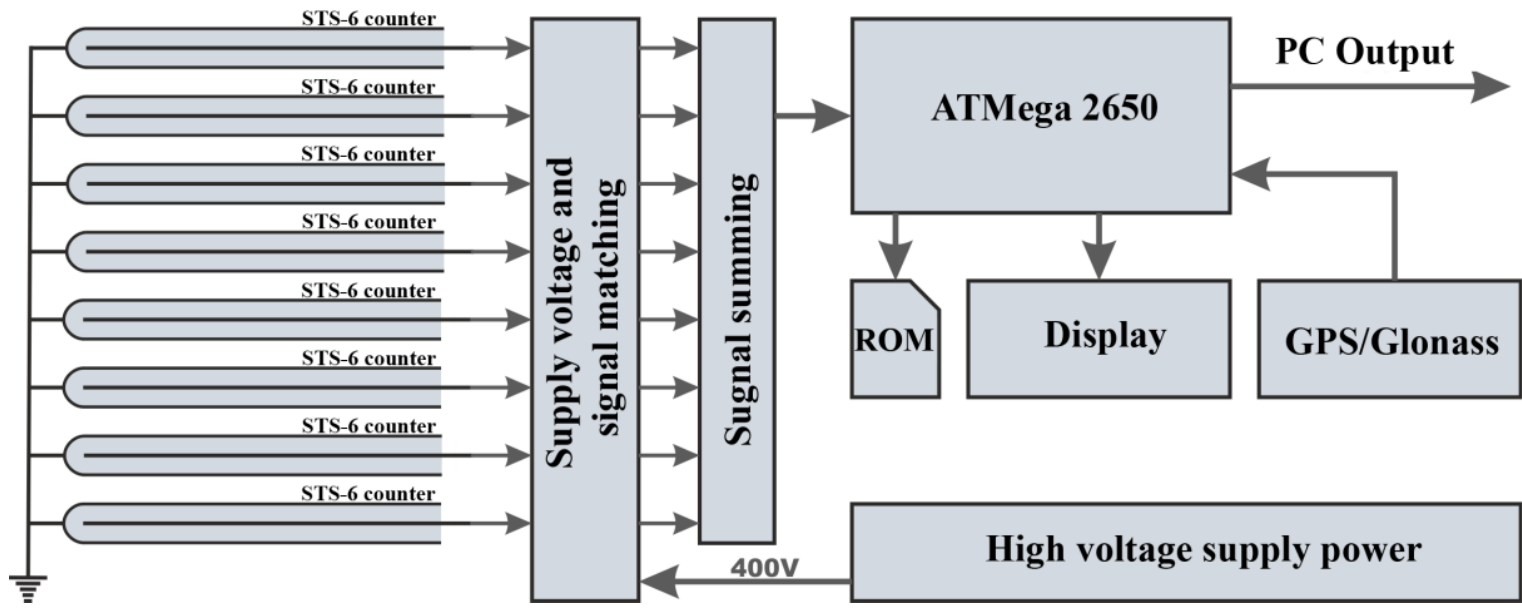
Apatity

Figure 8. Block diagram of a mobile detector of charged CR component, developed and commissioned at the CR station 

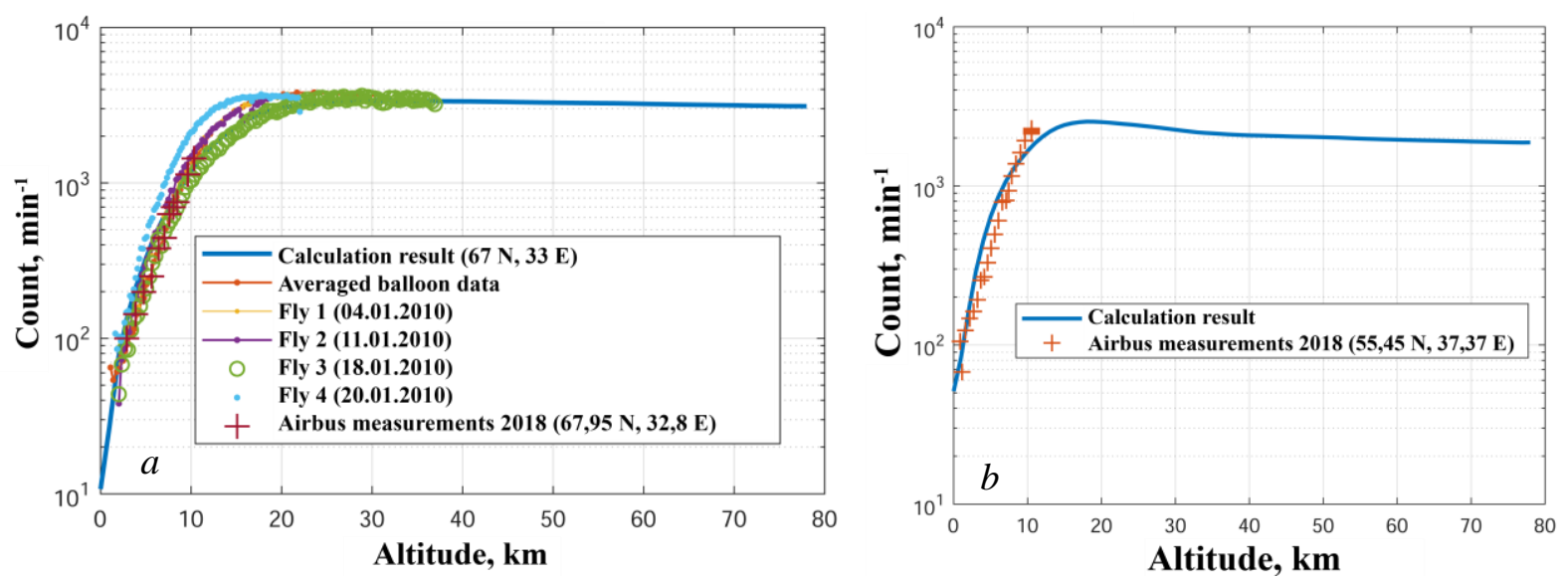

Figure 9. Comparison of vertical profiles of count rate obtained from balloons for different periods $(a)$ and from airplane measurements $(a, b)$ with the results of simulation of propagation of GCR protons for two points: Apatity $(a)$, Moscow $(b)$

\section{CONCLUSION}

We have calculated propagation of $\mathrm{CR}$ protons through Earth's atmosphere, using the improved RUSCOSMICS software package. Due to the optimization achieved by averaging physical parameters in describing geometry of the model and, consequently, by decreasing the number of logical volumes, we managed to obtain good agreement between count rate and correctness of the results, which was determined by comparing with experimental datasets. We are going to apply detectors of different types. Works on the simulation using parallel calculations are currently in progress. The data obtained can be of applied significance, for example, in assessment of the equivalent dose, and can be used in basic research, for example, for a detailed study of characteristics of cascades. All changes in releases, their descriptions, and archives of the obtained data are freely available on the website of the project [http://ruscosmics.ru].

The work was funded by the Russian Foundation for Basic Research under research project No.18-32-00626.

\section{REFERENCES}

Agostinelli S., Allison J., Amako K., Apostolakis J., Araujo H., Arce P., et al. Geant 4 - a simulation toolkit. Nuclear Instruments and Methods A. 2003, vol. 506, iss. 3, pp. 250-303. DOI: 10.1016/S0168-9002(03)01368-8.

Bazilevskaya G.A., Usoskin I.G., Flückiger E.O., Harrison R.G., Desorgher L., Bütikofer R., Krainev M.B., Makhmutov V.S., Stozhkov Y.I., Svirzhevskaya A.K., Svirzhevsky N.S., Kovaltsov G.A. Cosmic ray induced ion production in the atmosphere. Space Sci. Rev. 2008, vol. 137, iss. 1-4, pp. 149-173. 10.1007/s11214-008-9339-y.

GEANT4 Physics Reference Manual. URL: http://geant4userdoc.web.cern.ch/geant4-userdoc/UsersGuides/Physics

ReferenceManual/fo/PhysicsReferenceManual.pdf (accessed June 10, 2019).

GOST 25645.150-90. Luchi kosmicheskie galakticheskie. Model' izmeneniya potokov chastits [State Standard 25645.150-90. Galactic Cosmic Rays. Model of Particle Flux Variations]. Moscow, 1991, 11 p.

Maurchev E.A., Balabin Yu.V. RUSCOSMIC - the new software toolbox for detailed analysis of cosmic rays interactions with matter. Solar-Terrestrial Physics. 2016, vol. 2, no. 4, pp. 3-10. DOI: 10.12737/24269.
Maurchev E.A., Balabin Yu.V., Gvozdevsky B.B., Vashenyuk E.V. A new numerical model for investigating cosmic rays in the Earth's atmosphere. Bull. Russ. Acad. Sci. Phys. 2015, vol. 79, no. 5, pp. 657-659. DOI: 10.3103/ S1062873815050305.

Maurchev E.A., Mikhalko E.A., Germanenko A.V., Balabin Yu.V. The experimental methods for validating the results of modeling the cosmic rays interaction with the Earth's atmosphere. Trudy Kol'skogo nauchnogo tsentra RAN [Proc. of Kola Science Center RAS]. 2018, vol. 9, no. 5-4, pp. 76-81. DOI: 10.25702/KSC.2307-5252.2018.9.5.76-81. (In Russian).

Perez-Peraza J., Gallegos-Cruz A., Vashenyuk E.V., Balabin Yu.V., Miroshnichenko L.I. Relativistic proton production at the Sun in the October 28th, 2003 solar event. Adv. Space Res. 2006, vol. 38, iss. 3, pp. 418-424. DOI: 10.1016/j.asr.2005.01.082.

Perez-Peraza J., Vashenyuk E.V., Gallegos-Cruz A., Balabin Yu.V., Miroshnichenko L.I. Relativistic proton at the Sun in the 20 January 2005 solar event. Adv. Space Res. 2008, vol. 41, iss. 6, pp. 947-954. DOI: 10.1016/j.asr.2007.04.054.

Picone J.M., Hedin A.E., Drob D.P., Aikin A.C. NRLMSISE-00 empirical model of the atmosphere: Statistical comparisons and scientific issues. J. Geophys. Res. 2002, vol. 107, iss. A12, 1468. DOI: 10.1029/2002JA009430.

Stozhkov Yu.I., Svirzhevsky N.S., Bazilevskaya G.A., Kvashnin A.N., Makhmutov V.S., Svirzhevskaya A.K. Longterm (50 years) measurements of cosmic ray fluxes in the atmosphere. Adv. Space Res. 2009, vol. 44, iss. 10, pp. 1124 1137. DOI:10.1016/j.asr.2008.10.038.

Thébault E., Finlay C.C., Beggan C.D., Alken P., Aubert J., Barroiset O., et al. International Geomagnetic Reference Field: the 12th generation. Earth, Planets and Space. 2015, vol. 67, 79. DOI: 10.1186/s40623-015-0228-9.

Usoskin I.G., Desorgher L., Velinov P., Storini M., Flückiger E.O., Bütikofer R., Kovaltsov G.A. Ionization of the earth's atmosphere by solar and galactic cosmic rays. Acta Geophysica. 2009, vol. 57, no. 1, pp. 88-101. DOI: 10.2478/s11600-008-0019-9.

Vashenyuk E.V., Balabin Yu.V., Miroshnichenko L.I. Relativistic solar protons in the ground level event of 23 February 1956: New study. Adv. Space Res. 2008, vol. 41, pp. 926-935. DOI:10.1016/j.asr.2007.04.063.

Vashenyuk E.V., Balabin Yu.V., Gvozdevsky B.B. Features of relativistic solar proton spectra derived from ground level enhancement events (GLE) modeling. Astrophysics and Space Sciences Transactions. 2011, vol. 7, iss. 4, pp. 459-463. DOI: 10.5194/astra-7-459-2011. 
Velinov P.I.Y., Balabin Yu.V., Maurchev E.A. Calculations of enhanced ionization in strato-troposphere during the greatest ground level enhancement on 23 February 1956 (GLE 05). Compt. rend. Acad. bulg. Sci. 2017, vol. 70, no. 4, pp. 545-554.

URL: http://ruscosmics.ru (accessed June 10, 2019).

URL: http://ruscosmics.ru/CR.htm (accessed June 10, 2019).
How to cite this article

Maurchev E.A., Balabin Yu.V, Germanenko A.V., Mikhalko E.A., Gvozdevsky B.B. Calculating the ionization rate induced by GCR and SCR protons in Earth's atmosphere. Solar-Terrestrial Physics. 2019. Vol. 5. Iss. 3. P. 68-74. DOI: 10.12737/stp-53201908. 